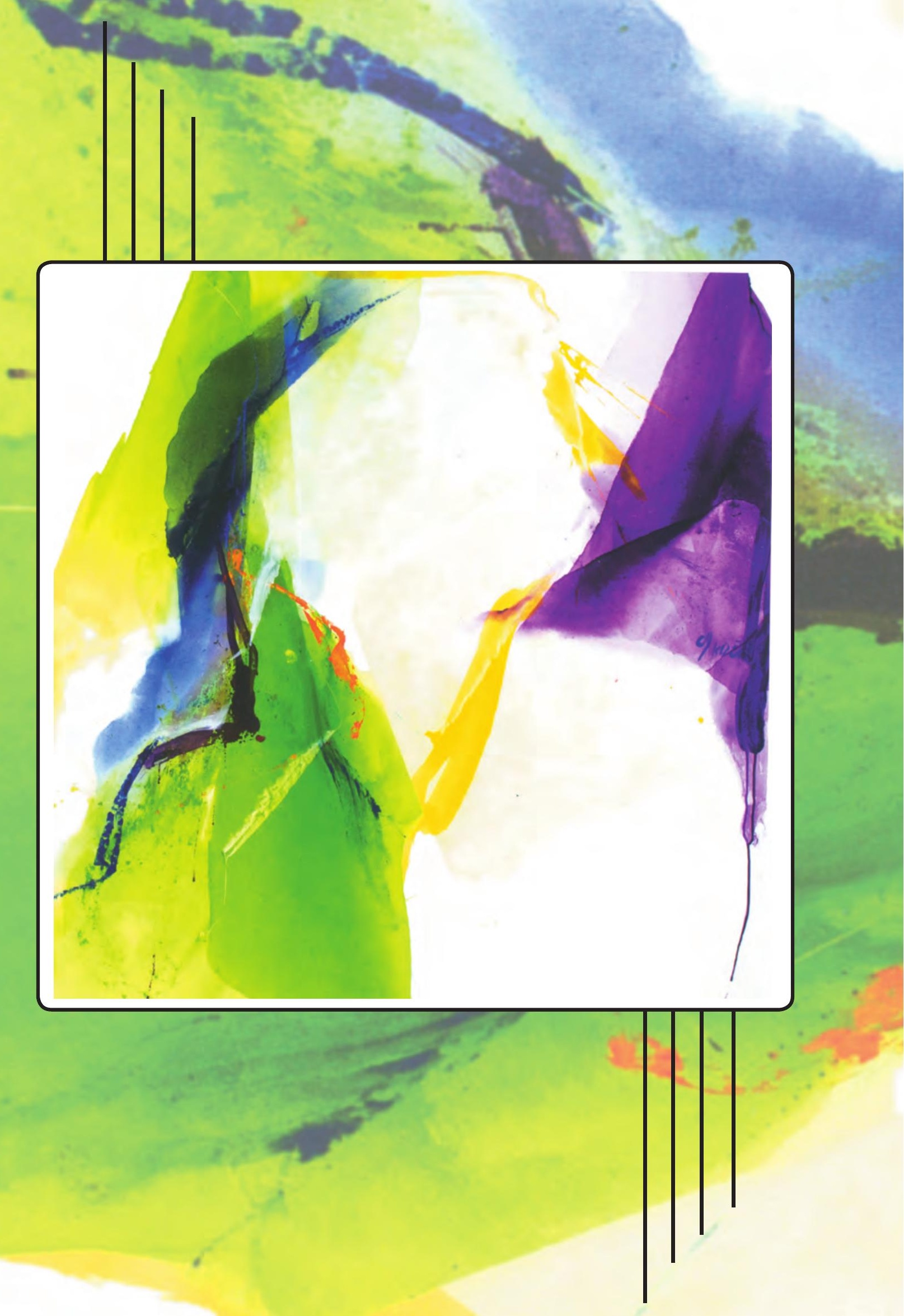


Hommage aux 12 apotres

Óleo sobre Lienzo

$120 \times 120 \mathrm{cms}$

2009

Colección Privada Universidad Santo Tomás 


\title{
TIPOLOGÍAS Y MODELOS DE CADENAS PRODUCTIVAS EN LAS MIPYMES
}

\author{
Lila Margarita Bada Carbajal \\ Luis Arturo Rivas Tovar ${ }^{2}$
}

\begin{abstract}
RESUMEN
Esta investigación describe las tipologías y modelos de cadenas productivas para las Micro, Pequeñas y Medianas Empresas (MIPYMES), reportadas en el estado del arte, y como principal aportación, ordena y crea categorías de análisis que facilitan la comprensión del complejo bosque teórico sobre el tema de la asociatividad de empresas. Tradicionalmente, en la literatura estratégica, los conceptos de cadenas productivas, cadenas del valor y clúster tienen fronteras difíciles de identificar. La principal aportación de este trabajo radica en la propuesta de una tipología y modelos sobre cadenas productivas. El enfoque de cadenas productivas es relativamente nuevo en los países latinoamericanos pero se usa desde hace décadas para orientar el trabajo en otros países principalmente europeos y es una herramienta de análisis que permite identificar los principales puntos críticos que frenan la competitividad de un producto o servicio de una empresa, para luego definir e impulsar estrategias centradas entre los principales actores involucrados.
\end{abstract}

\section{PALABRAS CLAVE}

Cadenas productivas, MiPymes, asociatividad.

\begin{abstract}
This research describes the typologies and models of productive chains for the Micro, Small and Medium Enterprises (MiPymes), which has been reported in the states of the art. The main contribution is that this research ordered and created analyzes of categories that has easy comprehension of the complex theoretical forest on themes about the associativity of enterprises. In the strategic literature traditional the concepts of productive chain, value chain and clusters have several limits that they are difficult to identify. The main contribution of this paper, is the proposal of a typology and model about productive chain. The focus of the productive chains is a related new concept in Latin-American countries but it has been used twenty years ago to direct the work. In Europe it's an analytical tool to aloud to identify critical factors that stop the competitiveness in products and services from different companies. After that it could be impulse strategies focused on the principal factors that affect the commodities chains.
\end{abstract}

\section{KEYS WORDS}

Productive chain, MIPYMES, asociativity

1 Doctorando en Ciencias Administrativas. Profesor categoría B, del Instituto Tecnológico Superior de Álamo Temapache Veracruz, México

2 Doctor en ciencias Administrativas. Profesor, Universidad Santo Tomás y Profesor invitado de la Universidad Politécnica de Madrid. Es miembro del Sistema Nacional de Investigación. Equipo de investigación del premio Nobel mexicano Mario Molina. 


\section{Introducción}

Las micro, pequeñas y medianas empresas (MiPymes) han demostrado, en diferentes tiempos y lugares, ser factor determinante del desarrollo económico de cada país, ya que siempre han sido elementos preponderantes en el caso de las economías en el término de empleo, inversión y producción.

La literatura administrativa sobre las MiPymes ha sido abordada desde distintas perspectivas, como lo es la competitividad, recursos administrativos, innovación, financiamiento y políticas de fomento, entre otras. La dimensión empresarial de las MIPYMES es un factor competitivo establecido por: la actividad económica, eficiencia del negocio, eficiencia del gobierno y la infraestructura (Marcolini, 2001). Los recursos generales con los que cuentan las MiPymes son humanos, recursos físicos, capital y capacidad (Taniguchi, 2006). En el campo de la innovación, la evidencia muestra que al mismo tiempo que se da una aceleración en los cambios tecnológicos, las MiPymes parecen jugar un papel pivote, en el proceso de desarrollo general (OECD, 1996).

En México las MIPYMES SON MUY RELEVANTES: el 99.9\% de nuestras empresas estaban compuestas por MiPymes, generaban el $79.6 \%$ del empleo y explicaban el 51.6 \% del PIB al año 2003. (Gaona - Aguirre, 2007).

a. Actualmente las economías que atraviesan un proceso de globalización implica: el desarrollo de las tecnologías de información (y de las comunicaciones), apertura comercial (reducción de barreras al comercio de bienes y servicios arancelarios y para arancelarios), procesos de integración regional (MERCOSUR, TLCAN, APEC, Unión Europea, ALCA, NAFTA), mayor flujo de capitales y desarrollo de los mercados financieros y transición del rol del Estado del paternalismo al papel promotor del desarrollo económico. Este proceso ha traído como consecuencia la creciente dificultad de las Micro, pequeñas y medianas empresas (MiPymes ) para competir en los mercados, principalmente porProblemas de información, tecnológicos, de financiamiento, de escasa articulación y de limitadas capacidades operativas y gerenciales.

b. Desventaja relativa en costos de distribución, producción, promoción entre otros, debido al escaso poder de negociación.

Debido a lo anterior una estrategia clave para el desarrollo de las MIPYMES en el mundo globalizado es fomentar la asociatividad de las empresas, al promover la creación de clústeres y redes empresariales en cadenas productivas competitivas.

La asociatividad es una estrategia resultado de una cooperación o coalición de empresas en función de un objetivo en común, en donde cada participante mantiene independencia jurídica y gerencial. (Laura M., 2006) 
Las formas de asociatividad son:

- Cadenas productivas

- Redes

- Clústeres

La definición de estos conceptos y su caracterización son el objeto de estudio de este trabajo.

Algunos beneficios de asociatividad son los siguientes (Dini, 2003):

- Economías de escala: de vital importancia para la MIPYME, para: el descuento en la compra de insumos, uso eficiente de las tecnologías productivas y acceso a los mercados más grandes.

- Flexibilidad: mayor capacidad de responder a los cambios en la demanda, sin aumentar capital instalado ni costos fijos.

- Difusión de la información: intercambio de conocimientos y experiencias para mejorar su capacidad de gestión estratégica y acelerar su aprendizaje.

- Menos barreras de entrada: al especializarse las empresas en las distintas fases de producción, se facilita la puesta en marcha de nuevos emprendimientos productivos.

- Pertinencia de las acciones de apoyo: mayor posibilidad de éxito de las acciones de apoyo, gracias a la fluida comunicación entre instituciones públicas y sector privado.

- Eficiencia de las acciones de apoyo.

\section{Método de investigación}

Es una investigación de tipo documental donde se realiza una búsqueda en las siguientes bases de datos:

- Proquest digital dissertation

- $\mathrm{ABI}$ Inform

- $\quad$ Esmerald

- Ocenet 
En esta investigación se analiza el concepto de cadena productiva, su utilidad, importancia, ventajas, procesos de formación y los tipos de cadena así como los modelos de cadenas productivas de valor los cuales tienen niveles: globales, y sectoriales.

Los sujetos de investigación son los tipos y modelos de cadenas de productivas en las MiPymes.

Como resultado de lo anterior se realizó un análisis de los modelos respaldados en el Estado del Arte.

\section{CADENA PRODUCTIVA}

\section{Estudios sobre cadenas productivas}

Los estudios sobre cadenas productivas tienen sus orígenes en Europa en los años setenta, permitieron mejorar la competitividad de varios productos como leche, carne y vino y promovieron la definición de políticas sectoriales consensuadas entre los diferentes actores de la cadena (Van Der Heyden D., 2006).

En Estados Unidos existen trabajos sobre cadenas productivas elaborados por el Banco Interamericano de Desarrollo de Washington D.C. (Pietrobelli, C., Rabelollotti, R. 2001 - Guaipatín C. 2004), donde alinean los factores claves que promueven la innovación: en educación (capacitación), acceso al crédito, existencia de instituciones efectivas y apertura económica. Así como, también establecen que para medir la capacidad de los países para renovarse constantemente y mejorar sus actividades productivas, es necesario que el país participe en las nuevas tecnologías e innovaciones. Así mismo en la Universidad de Duke, Durham, Carolina del Norte de Estados Unidos (Gereffi, G.2005), la Universidad de Ohio (Coxton, K., García - Dastugue, S., Lambert, D. 2001) y la Universidad de Nevada en Reno (Rogers, D. 2001) han realizado investigaciones sobre cadenas productivas para estudiar las múltiples dimensiones de ascenso industrial, el cual constituye una nueva forma de análisis del desarrollo económico en la era de una industrialización orientada a las exportaciones, donde se establecen implicaciones teóricas para el desarrollo del enfoque de las cadenas productivas con bases históricas y organizativas.

Las cadenas productivas han servido en la actualidad como una base importante de cualquier política industrial y han demostrado que sobre ellas se puede construir una política de desarrollo articulada, tal es el caso de los países latinoamericanos donde el enfoque de cadenas productivas es relativamente nuevo y, a partir del año 2000, se han realizado estudios de cadenas productivas en: Colombia, Perú, Bolivia, Costa Rica, Brasil, Argentina, Venezuela y México, donde, a través de sus instituciones educativas de posgrado e investigación y gubernamentales han 
demostrado que el enfoque de cadena productiva es pertinente en el contexto actual de evolución de la economía mundial, competitividad, productividad, globalización, innovación tecnológica y complejos sistemas agroalimentarios por lo cual el enfoque permite dar una mirada sistemática a las actividades productivas.

En México se han realizado estudios de cadenas productivas a partir del 2002, a través de instituciones gubernamentales como S.E., SAGARPA, SPYME, NAFIN, INIFAB, PECYTAJ y el Consejo Nacional para la Competitividad de la Micro, Pequeña y Mediana Empresa, que han permitido el establecimiento de planes sectoriales y regionales y, programas de competitividad para incrementar la competitividad y productividad de las MIPYMES a través de la integración en cadenas productivas. Así como también instituciones educativas como el Instituto Tecnológico de Estudios Superiores de Monterrey (2004)33, la Universidad de Aguascalientes (Carranza R., Valvidia A. 2005) ${ }^{4}$ y la Universidad de Chapingo (Cuevas, V. 2006) $)^{5}$, han realizado investigaciones que tienen como objetivo promover la movilización de recursos productivos existentes en las zonas rurales, a través de una mejor vinculación de los pequeños productores en las cadenas productivas, con el fin de analizar el desempeño de la cadena productiva, e identificar sus factores críticos y potenciales de desarrollo.

\section{Concepto de cadena productiva}

De acuerdo con la CEPAL 2003, el concepto de cadenas productivas implica la concentración sectorial y/o geográfica de empresas que desempeñan las mismas actividades o actividades estrechamente relacionadas entre sí (tanto hacia atrás como hacia delante) con importantes y acumulativas economías externas y posibilidad de llevar a cabo una acción conjunta en la búsqueda de la eficiencia colectiva.

La idea que subyace al concepto de cadenas productivas es que la eficiencia de la cadena es mayor a la que obtendría cada empresa en el caso de que trabajara de forma independiente, por lo tanto, genera beneficios para todas las empresas que se encuentran en cadena.

Este enfoque de cadena productiva, desarrollado en Europa en los años setenta, ha permitido mejorar la competitividad de los productos y servicios de las empresas al promover la definición de políticas sectoriales consensuadas entre los diferentes actores de la cadena.

Estudios sobre cadenas productivas para la seguridad alimentaria y desarrollo rural.

Estudio sobre cadena productiva de leche del Estado de Aguascalientes.

Estudio sobre cadena productiva de leche de vaca del Estado de Hidalgo. 
Figura 1: Cadena productiva

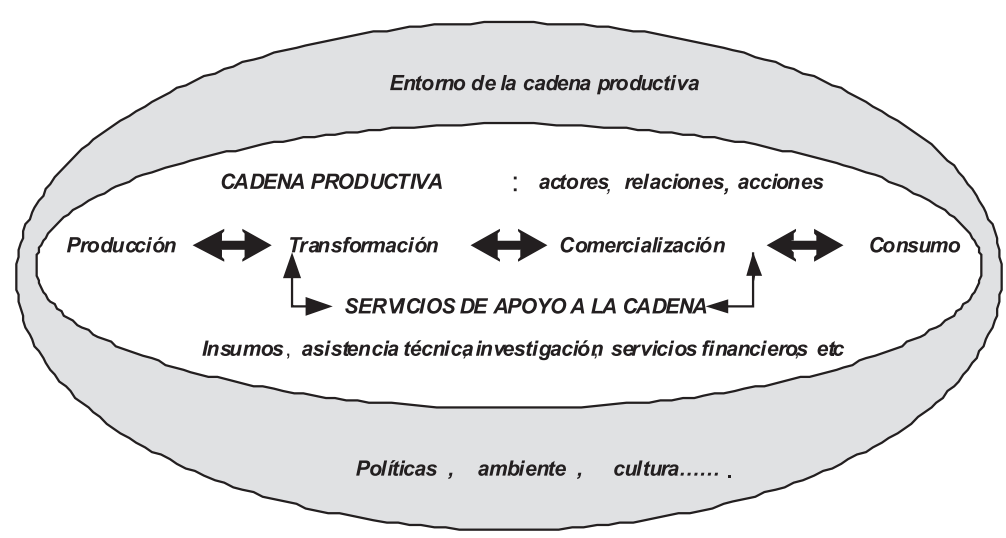

Fuente: Guía Metodológica para el análisis de cadenas productivas, Van Der Heyden y Camacho 2006

La Figura 1, muestra la cadena productiva donde se encuentran presentes actores y trabajos diferenciados alrededor de los productos o servicios que ofrecen las empresas. Estos actores se vinculan entre sí para llevar el producto o servicio de un estado a otro, desde su producción hasta el consumo. La estructura dinámica de todo este conjunto de actores, acciones, relaciones, transformaciones y productos o servicios es lo que se conoce como cadena productiva (Kaplinsky, 2000).

Desde el punto de vista legal, el Diario Oficial de la Federación en la Ley para el Desarrollo de la Competitividad de las MiPyme 2002, define las cadenas productivas como:

"Sistemas productivos que integran conjuntos de empresas que añaden valor agregado a productos y servicios a través de las fases del proceso económico”.

Enfocándose en incrementar la participación de las MiPyme en los mercados, en un marco de crecientes encadenamientos productivos que generen mayor valor agregado nacional; con la finalidad de fomentar el empleo y el bienestar social y económico de todos los participantes en las MiPyme.

\section{Utilidad de la cadena productiva}

La idea de una cadena productiva está centrada en las actividades necesarias para convertir la materia prima en productos terminados y venderlos $\mathrm{y}$, del valor que se le agrega en cada eslabón (Gereffi, 1999). 
El diseño, la producción y la comercialización de productos de una empresa implica una cadena de actividades repartidas entre diferentes empresas, a menudo localizadas en diferentes lugares y países, para formar las cadenas productivas.

De acuerdo con Kaplinsky (2000) y Wood (2001), la cadena productiva desde el punto de vista analítico es útil por tres razones principales:

1. El enfoque se desplaza de la fabricación a las otras etapas que comprende el suministro de bienes y servicios a los consumidores, se presta más atención en las etapas "intangibles", tales como la distribución y la comercialización.

2. Genera flujos de información entre las etapas de actividad de la cadena al acentuar que las relaciones entre las empresas no siempre son en condiciones de igualdad e implican competencia.

3. La clave para comprender la apropiación global de los retornos de la producción es la habilidad de identificar actividades de alto rendimiento dentro de la cadena productiva.

Para realizar un análisis de las cadenas productivas se debe de enfocar en las relaciones de los actores que participan en la cadena y sus implicaciones para el desarrollo. En cualquier punto de la cadena, se necesita algún grado de gobierno o coordinación para determinar qué, cómo, cuánto se produce.

Humphrey y Schmitz (2000) distinguen tres tipos de gobierno o coordinación:

a. Redes: cooperación entre empresas de más o menos el mismo poder y que comparten sus competencias dentro de la cadena.

b. Cuasi-jerárquica: relaciones entre empresas jurídicamente independientes donde una está subordinada de la otra y donde un líder en la cadena determina las reglas que el resto de los actores deben cumplir.

c. Jerárquica: donde una empresa es propiedad de otra empresa externa.

\section{Importancia de las cadenas productivas}

Las cadenas productivas involucran todos los eslabones de la actividad desde los fabricantes de insumos, maquinaria y equipos, hasta el producto final, sin dejar de lado la parte de la comercialización, dado que el consumidor se constituye en el último eslabón.

La cadena identificada permite localizar los productos, procesos, las empresas, las instituciones, las operaciones, las dimensiones y las capacidades de negociación, las tecnologías y las relaciones de producción. 
Algunos puntos que resaltan la importancia de las cadenas productivas son los siguientes:

- La competitividad de los países de Europa y Norte América se basa en la especialización, la división del trabajo y la optimización de las cadenas de valor.

- Las empresas modernas continuamente redefinen sus competencias clave: profundizan aquellas actividades en las que tienen ventajas competitivas y se retiran de otras actividades en las que no hay competencias claras.

- Al mismo tiempo intensifican la cooperación con empresas que las proveen de subproductos y servicios complementarios. (García, 2005)

\section{Ventajas de las cadenas productivas}

En una economía globalizada, como la mexicana, la elaboración de estrategias de desarrollo debe considerar el concepto de cadena productiva que se refiere a un producto o a un grupo de productos conjuntos o ligados para el uso.

Desde este punto de vista las ventajas en la cooperación en cadenas productivas son:

- La especialización es un estabón de la cadena productiva que permite dedicar los recursos a lograr la excelencia de una actividad muy específica.

- La combinación de varias empresas altamente especializadas resulta en cadenas muy productivas y competitivas.

- La estrecha cooperación con otras empresas de la cadena acelera el ritmo de innovaciones. Muchas innovaciones surgen a través de la búsqueda conjunta de soluciones entre proveedor y cliente.

El desarrollo de las cadenas productivas trae consigo una serie de externalidades positivas que permiten afrontar el entorno regional caracterizado por la elevada presión competitiva, (Pierce, 2003).

De acuerdo a la Universidad Agraria La Molina de Perú (2005, p.1), las ventajas de la integración en una cadena productiva son las siguientes:

- El fortalecimiento de las instituciones participantes en los acuerdos

- Mayor rentabilidad en la producción al obtener mejores precios y reducir los costos

- Disminución del riesgo

- Facilidad en el acceso a los insumos

- Acceso a fuentes de financiamiento, créditos y economías de escala 
- Acceso a información de mercados

- Mayor acceso a tecnologías de punta

- Mejor aprovechamiento de la mano de obra familiar

\section{TIPOS DE CADENAS PRODUCTIVAS}

De acuerdo con Villacorta (2005); los tipos de cadenas productivas basados en los componentes que las integran son los siguientes:

1. Cadena completa. Es una cadena productiva, compuesta por todos los elementos (proveedores de insumos, sistemas productivos, agroindustria, comercialización, mayorista y minorista y consumidores finales).

2. Cadena incompleta. Es una cadena productiva con uno o más de los componentes anteriores.

3. Cadena integrada. Es una cadena productiva cuyo producto se constituye en insumo para otra cadena.

La cadena productiva es el proceso que sigue un producto o servicio a través de las actividades de producción, transformación e intercambio, hasta llegar al consumidor final. Incluye, además, el abasto de insumos (financiamiento, seguros, maquinaria, equipo y materias primas directas e indirectas, entre otros) y sistemas relevantes, así como todos los servicios que afectan de manera significativa a dichas actividades: investigación, desarrollo y asistencia técnica entre otros (Programa Estatal de Ciencia y Tecnología del Estado de Jalisco 2007).

\section{MODELOS DE CADENAS PRODUCTIVAS}

La información es un aspecto fundamental de cada una de las etapas de la cadena. En la Figura 2, se aprecia el modelo de la vieja cadena productiva, secuencial, estática y analógica, que ha sido reemplazada por una nueva cadena no lineal, dinámica, colaborativa, sistemática y estable. El valor agregado se basa en la conversión de datos en información, la información en conocimiento y el conocimiento en producto de alto valor.

De acuerdo al Programa Estatal de Ciencia y Tecnología del Estado de Jalisco, México. (2007, p.50), establece que las escalas y, por ende, la orientación de las cadenas productivas dependen de los principales factores de operación identificados por los modelos de competitividad de la MiPyme: tecnología, capital y mano de obra, entiéndase ésta como capacitación del recurso humano y organización del mismo 
dentro de la empresa. Así que el factor tecnológico asociado a la formación del recurso humano, se convierte en elemento clave de integración de cadenas productivas y de la generación de valor.

Figura 2: Modelo de la vieja cadena productiva vs. la nueva cadena productiva.

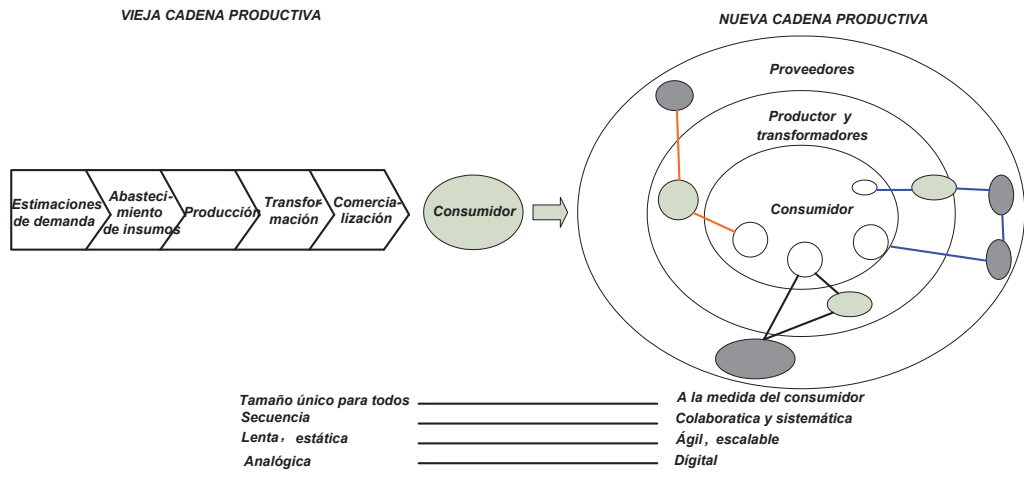

Fuente: Programa Estatal de Ciencia y Tecnología del Estado de Jalisco 2007.

Los modelos de cadenas productivas son globales y sectoriales. De acuerdo a la revisión de la literatura con base en las cadenas productivas, se presentan los diferentes modelos, globales de Gereffi (1999) y los sectoriales: agropecuario, industrial y de servicios de García (2005) y Campebell (1999).

\section{a) Modelos de cadenas productivas globales}

La literatura sobre cadenas productivas globales (Gereffi, 1999) daría entender que la cuasi jerarquía donde los fabricantes y compradores desempeñan el papel principal, domina el grupo de las manufacturas tradicionales. En algunos casos coexisten cadenas productivas diferentes, con empresas que participan tanto en una cadena productiva local como en una global.

El capital industrial y el comercial han promovido la globalización, al establecer tipos diferentes de redes económicas internacionales, que puedan denominarse respectivamente, cadenas productivas dirigidas al productor y cadenas productivas dirigidas al comprador (Gereffi 1994 y 1999). 
El estudio del Programa de Ciencia y Tecnología de Jalisco, establece que una cadena productiva (commodity chain), se refiere al amplio rango de actividades involucradas en el diseño, producción y comercialización de un producto $(2007$, p.50).

\section{Cadenas productivas destinadas al productor}

Las cadenas productivas dirigidas al productor (Figura 3), son aquellas en las que los grandes fabricantes, comúnmente transnacionales, juegan los papeles centrales en la coordinación de redes de producción (incluido vínculos hacia atrás y hacia delante), (Gereffi, 2001, p.15).

Esto es característico en industrias de capital e industrias con tecnología intensiva, tales como las automotrices, las de aviones, computadoras, semiconductores y maquinaria pesada. La industria automotriz constituye una ilustración clásica de una cadena dirigida al productor, con sistemas de producción multilaterales que involucran a miles de empresas.

Figura 3: Cadena productiva destinada al productor

Fuente: Problemas de Desarrollo. Universidad Autónoma de México, Gereffi; 2001

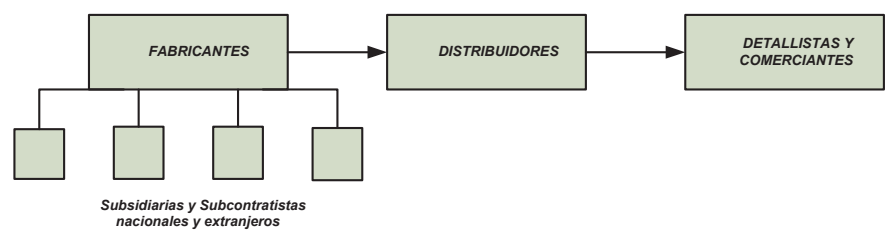

\section{Cadenas productivas destinadas al comprador}

Las cadenas productivas destinadas al comprador (Figura 4), se refiere a aquellas industrias en las que los grandes detallistas, los comercializadores y los fabricantes de marca juegan papeles de pivotes en el establecimiento de redes de producción descentralizada en una variedad de países exportadores, comúnmente localizados en el tercer mundo. (Gereffi; 2001).

El estudio de de Gereffi establece que este modelo de industrialización dirigida al comercio se ha hecho común en las industrias de artículos para el consumidor que cuentan con una fuerza de trabajo intensiva, tales como la del vestuario, zapatos, juguetes, artículos para el hogar, electrónica y de una variedad de artesanías. La producción generalmente la llevan a cabo en redes de contratistas de Tercer Mundo que realizan artículos terminados para compradores extranjeros. Las especificaciones 
son suministradas por los grandes mayoristas o comerciantes que ordenan los artículos. Gereffi (2001, p.16).

Una de las características principales de las empresas que se ajustan al modelo dirigido al comprador, incluidos detallistas tales como Wal. Mart. Sears Roebuck y J.C. Penney, compañías de calzado deportivo como Nike y Reebok, y empresas orientadas a la moda como Liz Clairborne, The Gap y The Limited, que diseñan y/o comercializan, pero no fabrican, los productos de marca que ordenan. Forman parte de una nueva clase de fabricantes sin fábrica (manufactures without factories) que en el proceso de producción separan la producción física de artículos de la etapa del diseño y la comercialización (Gereffi, 1994).

Comúnmente las empresas de las cadenas destinadas al productor pertenecen a oligopolios globales. En contraste, las cadenas de productos destinados al comprador se caracterizan por una alta competitividad y sistemas de fábricas globalmente descentralizadas.

Figura 4: Cadena productiva destinada al comprador

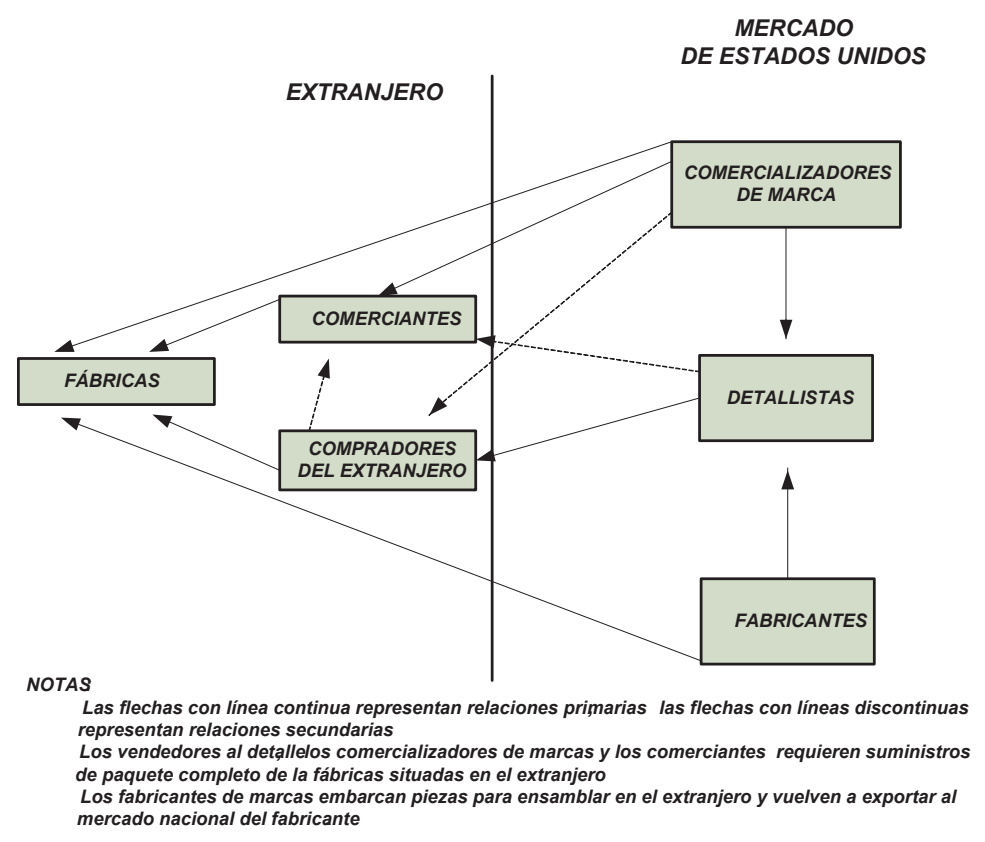

Fuente: Problemas de Desarrollo. Universidad Autónoma de México, Gereffi; 2001 


\section{b) Modelos de cadenas productivas por sectores económicos}

Estos modelos de cadenas productivas están integrados por los tres sectores económicos de la economía mexicana: el sector agropecuario, industrial y de servicios. Estos sectores tienen relaciones intersectoriales debido a que el sector agropecuario le vende materias primas agrícolas al sector industrial y le compra fertilizantes, abonos, y maquinaria agrícola. El sector servicios le compra alimentos al sector agropecuario, éste le solicita servicios financieros, comerciales y de transporte al sector servicios. El sector industrial le vende al sector servicios, muebles, equipo de oficina y camiones entre otros; el sector servicios le proporciona a la industria servicios profesionales, médicos, financieros otros.

\section{Cadenas productivas del sector agropecuario}

El sector agropecuario antes llamado sector primario, se encuentra formado por cuatro ramas o actividades económicas: agricultura, ganadería, silvicultura y pesca.

Los sectores agropecuarios presentan una falta de integración entre los agentes productivos, los cuales por el contrario compiten individualmente entre sí (proveedores de insumos, intermediarios y medios de transformación, comercialización entre otros), (García, 2005).

A continuación se detallan los modelos de cadenas productivas de las cuatro actividades que conforman este sector.

\section{Cadenas productivas de actividades agrícolas}

La agricultura es el arte de cultivar la tierra. Las actividades económicas que abarca el sector agrícola, tienen su fundamento en la explotación del suelo o de los recursos que éste origina en forma natural o por la acción del hombre: cereales, frutas, hortalizas, pasto, forrajes y otros variados alimentos vegetales. La agricultura es una actividad de gran importancia estratégica como base para el desarrollo autosuficiente y riqueza de las naciones.

García, J. (2003), propone el modelo de las tres estructuras posibles de las cadenas productivas agrícolas (Figura 5), donde en la primer estructura muestra una cadena productiva en un sólo sentido, los actores que la forman van desde el productor hasta el consumidor final y el producto se destina sin transformación alguna; en la segunda estructura, la cadena productiva tiene implícita la relación a la agroindustria rural en donde el producto puede llevarse al consumidor final en su versión original y con un proceso de transformación implícito, en la tercera estructura, la cadena productiva contiene el eslabón de la asociación de productores donde destina el producto ya sea 
en su versión original o procesado para el minorista o para la exportación y existen dos tipos de consumidores finales el local y el externo. Estás estructuras dependen de la cantidad de producción y de la organización de los actores.

Figura 5: Tres estructuras posibles de las cadenas productivas agrícola

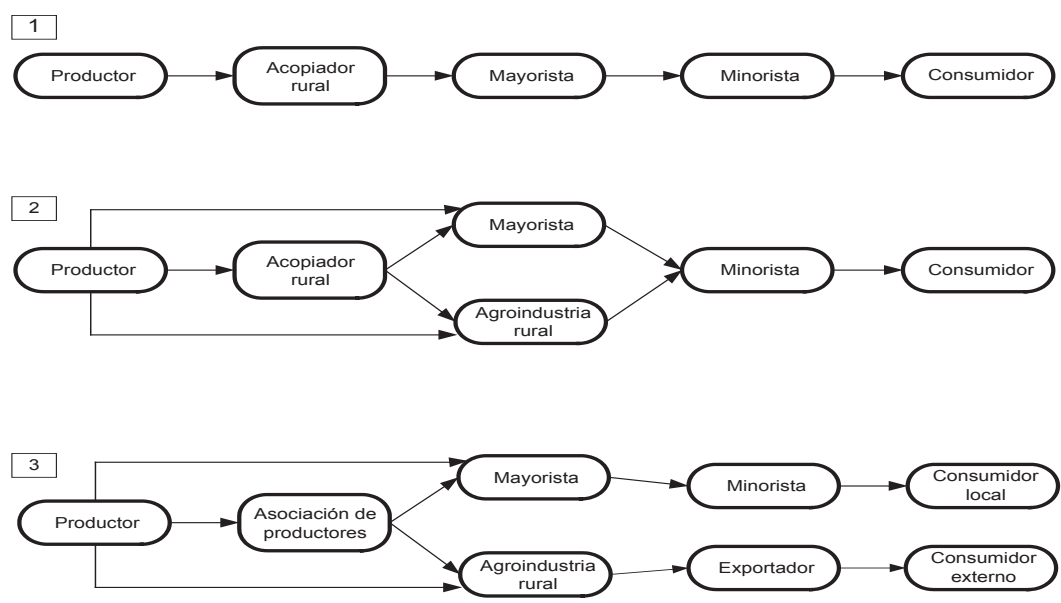

Fuente: García, J. MiPyme, clústeres y cadenas productivas, Universidad de Lima 2005

\section{Cadena productiva de actividades ganaderas}

La ganadería es una actividad económica, dedicada a la crianza del conjunto de especies animales para sacar provecho al animal y a sus productos, así como la propia explotación del ganado. La ganadería tiene como objetivo la producción de animales para obtener carne y derivados, como la leche, cuero, lana. La ganadería bovina, porcina, equina, caprina y ovina son las más comunes, pero últimamente la cría de liebres, cuyes, carpinchos, nutrias y otros animales alternativos ha comenzado a aumentar.

En la Figura 6, se aprecia el modelo de la cadena productiva de ganado caprino en Bolivia propuesto por Valdivieso, F. et, al. (2006) presenta el enfoque sistémico en la relación entre actores sociales e instituciones públicas y privadas de apoyo al sector caprino, así como el análisis global de las variables, al realizar un análisis 
crítico y reflexivo de la complejidad de sus componentes. Del estudio de este modelo se puntualizó que la leche de cabra tiene muchas propiedades nutritivas, proteicas y bajo contenido de grasas, por lo que la demanda de este producto se encuentra en ascenso, los derivados lácteos no son los únicos generadores de ingreso también se puede dar valor agregado al cuero, carne, y al guano, entre otros. Por tanto, la crianza de este ganado contribuye aproximadamente un $42 \%$ al ingreso familiar.

El ambiente institucional en el que se desenvuelve la cadena productiva de caprinos, está relacionado con dos organizaciones: la Secretaría de Promoción del Desarrollo Económico Local Agropecuario Potosí (SP-DELAP), que dispone de recursos financieros para asistencia técnica (AT) en los tres eslabones y apoyo financiero (AF) en transformación y comercialización. El Centro Educativo Técnico Agropecuario Mosoj Llanta (CETA), proporciona asistencia técnica (AT) a los eslabones de producción y transformación. Los Gobiernos Municipales apoyan indirectamente, proporcionan infraestructura productiva (sistemas de riego) y caminera (caminos vecinales); las fundaciones (FDTA valles y FDTA altiplano) no apoyan a la cadena productiva de caprinos.

\section{Figura 6: Cadena productiva de ganado caprino en Bolivia}

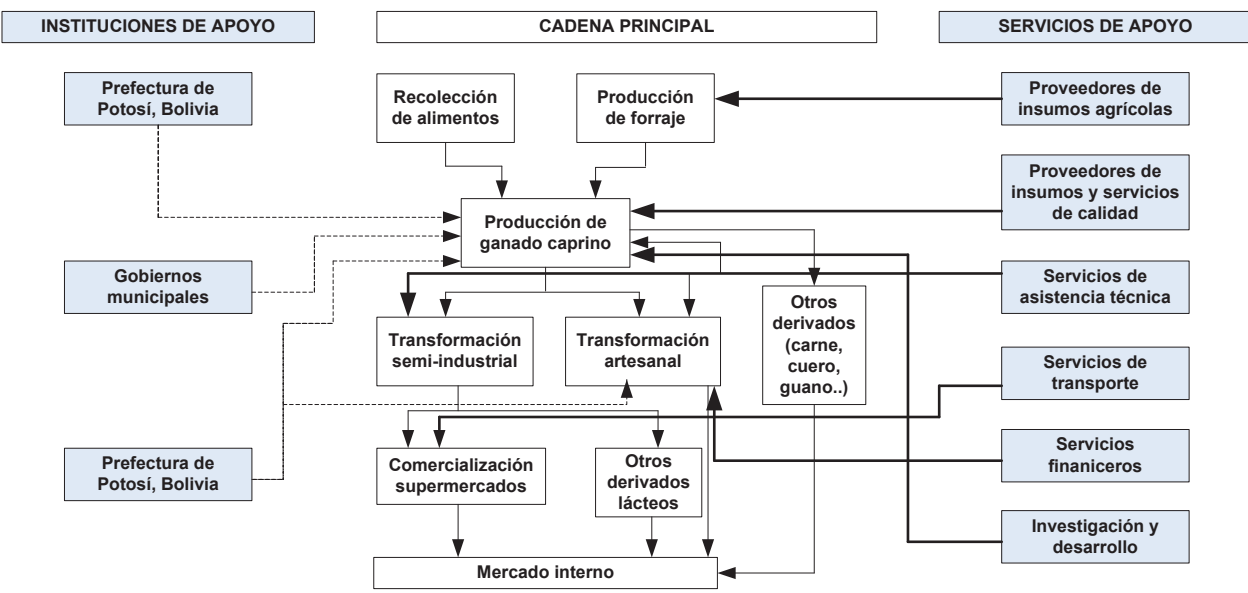

Fuente: Estudio-prospección de la cadena productiva de caprinos, en el área de intervención de la Secretaría de Promoción de Desarrollo Económico Local Agropecuario Potosí, Bolivia (2006). 


\section{Cadena productiva de actividades silvícolas}

La silvicultura es el cultivo de los bosques, selvas o montes y también, por extensión, la ciencia que trata de este cultivo, es decir, de las técnicas que se aplican a las masas forestales para obtener de ellas una producción continua de bienes y servicios demandados por la sociedad.

Derás, J. (2003), establece el modelo de la cadena productiva del bambú de Costa Rica, (Figura 7), donde en la dimensión horizontal al interior de cada eslabón predomina la acción individual de los actores y la dispersión geográfica, no hay mecanismos de articulaciones entre los actores, ni de comunicación, intercambio o cooperación. En la dimensión vertical a lo largo de la cadena se dan interacciones entre los diferentes eslabones que no van más allá de un traspaso de materia prima de productos de un eslabón a otro. Los costos de transacción de la cadena del bambú son elevados y afectan la eficiencia de la cadena productiva, limitan el quehacer de los productores, intermediarios, transformadores, distribuidores y consumidores y disminuye la competitividad de la cadena. No obstante la cadena productiva del bambú en Costa Rica, tiene el potencial que le permitiría desarrollarse competitivamente ya que existe un polo de desarrollo del bambú en una provincia de Costa Rica (Pérez Zeledón) donde se procesa el $67 \%$ de la materia prima a nivel nacional y se podría aplicar un enfoque territorial para el desarrollo de un clúster de pequeña y mediana industria.

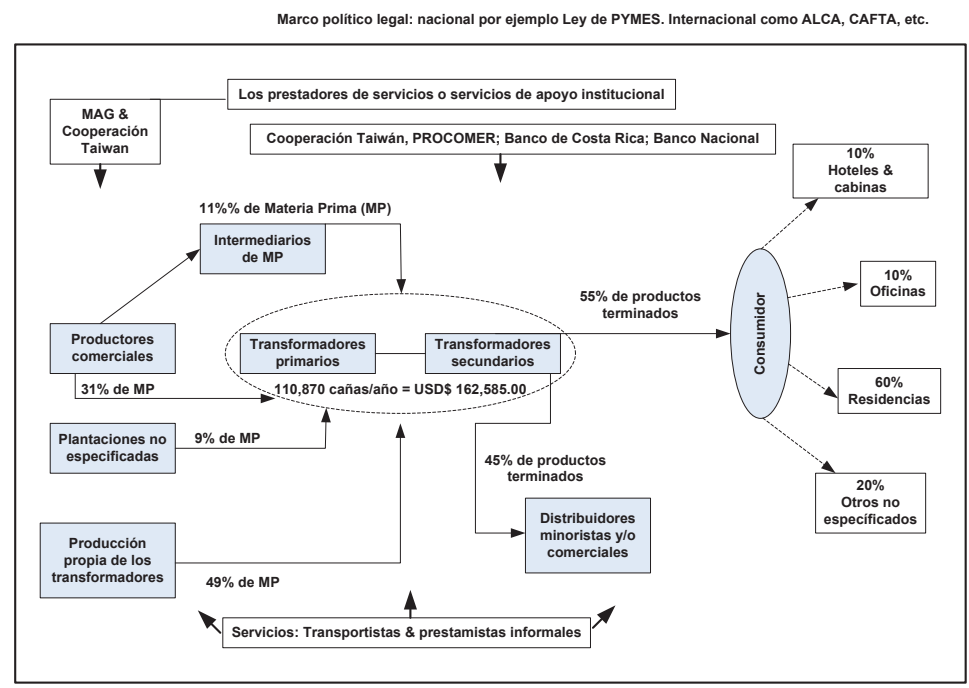

Figura 7: La cadena productiva de bambú en Costa Rica 
Fuente: Derás, J. (2003). La cadena productiva del bambú en Costa Rica

\section{Cadena productiva de actividades pesqueras}

La pesca es la acción de sacar o tratar de sacar los peces u otras especies animales útiles al ser humano, del agua. Las actividades económicas de pesca y acuicultura animal son realizadas por unidades pesqueras o acuícolas (personas físicas, cooperativas, uniones y otras) dedicadas principalmente a la captura, extracción o acuicultura de especies acuáticas con carácter comercial o en fomento, en el mar, ríos, lagos, lagunas, esteros, presas y estanques, entre otros cuerpos de agua (INEGI, 2004).

Gomes De Castro A, (2003), propone un modelo de cadena productiva de la Costa Pacífica Sudamericana (Colombia, Ecuador, Perú y Chile), (Figura 8), dicho modelo toma en cuenta el nivel de profundidad de información, la cuestión de sustentabilidad de los recursos naturales que soportan la actividad pesquera y la competitividad de los productos de las cadenas productivas del complejo agroindustrial de la pesca en los cuatro países. Este modelo tiene por objetivo la identificación de factores críticos limitantes al desempeño actual de la cadena, a través de análisis de flujos de materiales y de capital, de procesos productivos, de entradas y salidas en cada subsistema (eslabones o segmentos) y de las interacciones entre eslabones y segmentos. Los factores críticos identificados corresponderán a las demandas actuales (tecnológicas o no-tecnológicas) de la cadena productiva de pesca en el país.

Este modelo comprende 8 etapas:

1. Definición de los objetivos y de los límites del estudio de la cadena productiva de pesca de cada país

2. Caracterización del mercado consumidor de la cadena

3. Construcción de un modelo específico de la cadena productiva de pesca

4. Establecer las relaciones entre los segmentos (actores sociales) de cadena productiva, en términos de flujos físicos de productos y de capital

5. Analizar los flujos físicos, y definir los principales productos y características de calidad

6. Analizar los procesos productivos de los principales actores de la cadena productiva, en relación a la eficiencia, calidad y sostenibilidad ambiental

7. Identificación de limitantes y oportunidades

8. Identificación y priorización de factores críticos de desempeño. 
Figura 8: Cadena productiva de pesca de la Costa Pacífica Sudamericana

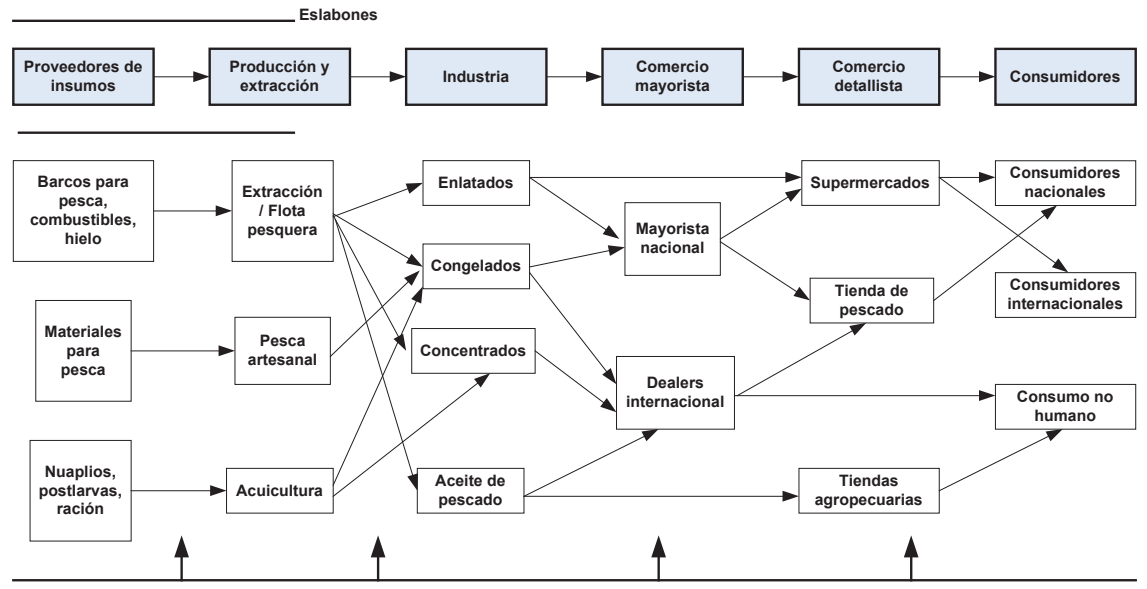

Ambiente organizacional e institucional (I\&D, asistencia técnica, crédito, transporte, impuestos, leyes ambientales, barreras no tarifarias, etc.)

$=$ Flujo de material

Fuente: Gomes De Castro, A. (2003). ONUDI. Foresight study on the productive chain of the fishery industry in the region of the South American Pacific Coast

\section{Modelos de cadenas productivas agroindustriales}

Los modelos de cadenas productivas agroindustriales tienen relaciones intersectoriales con las actividades agropecuarias e industriales. Las cadenas productivas agroindustriales, demuestran la importancia de la tecnología en el acercamiento del productor al consumidor final, vía tecnología desde el punto de vista de venta, apoyadas fuertemente en tecnologías de información (Programa Estatal de Ciencia y Tecnología del Estado de Jalisco, 2007).

En la Figura 9, se aprecia el modelo de cadena productiva agroindustrial, de Gomes De Castro, (2002); está compuesto por conjuntos de actores sociales interactivos, tales como sistemas productivos agropecuarios, proveedores de servicios e insumos, industrias de procesamiento y transformación, distribución y comercialización, además de consumidores finales del producto y subproductos de la cadena. Los actores sociales de cada cadena productiva pueden presentar un comportamiento cooperativo o conflictivo entre sí, en situaciones diversas. 
Figura 9: Cadena productiva agroindustrial

AMBIENTE INSTITUCIONAL

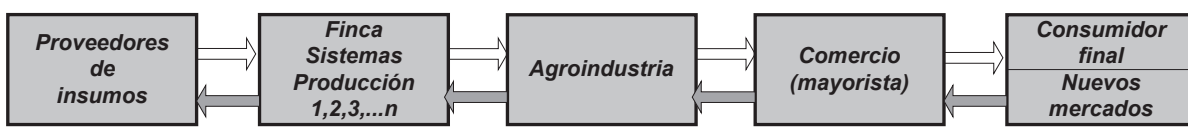

AMBIENTE ORGANIZACIONAL

$\square$ Flujos de materiales $\quad$ Flujos de capitales

Fuente: Productive chain: a conceptual frame for supporting technological prospection.

Gomes De Castro, 2002

La Figura 10 muestra la cadena productiva de la agroindustria del café, donde se específica la ruta más común que presenta su comercialización, además muestra que en la mayoría de los casos el potencial de valor agregado por parte de los productores es mucho más significativo que el que puede agregar al sector comercio. Así mismo, dado que el 90\% del café se comercializa a través de intermediarios, representa un área de oportunidad importante para que el productor primario pueda simplificar y participar en este proceso, a fin de apropiarse de una parte de valor que genera el mismo.

Figura 10: Cadena productiva agroindustrial del café

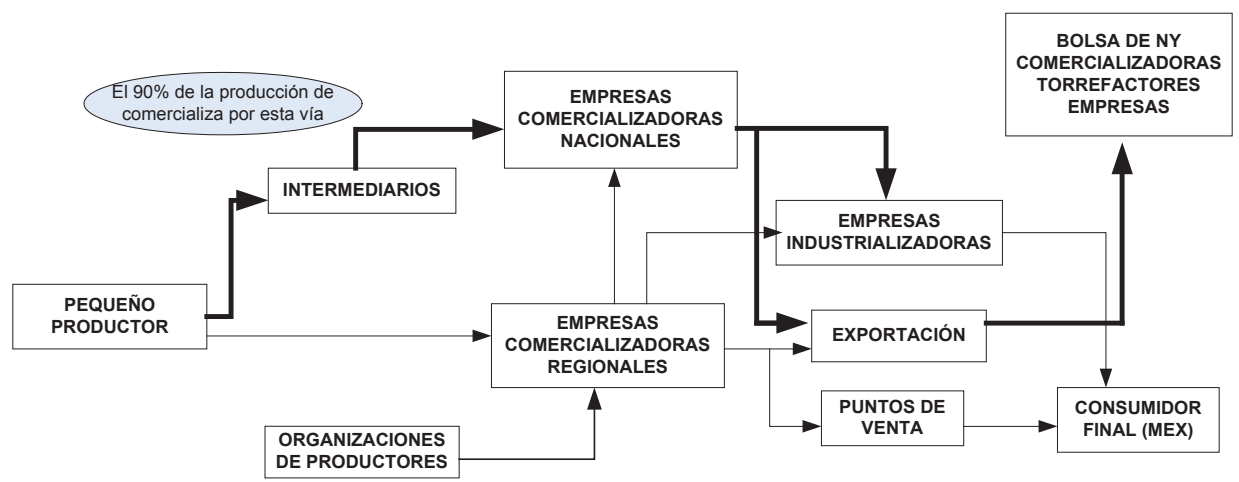

Fuente: SAGARPA 2006 


\section{Modelos de cadenas productivas del sector industrial}

El sector industrial tradicionalmente llamado sector secundario de la economía, se divide en dos subsectores: industria extractiva e industria de transformación.

Las cadenas productivas industriales muestran el valor más significativo en la calidad del proceso de transformación del producto y la tecnología así como también en la distribución y comercialización de los productos (Campebell J, Rozemberg, R. y Svarzman, 1999).

En la Figura 11, se muestra la cadena productiva textil y confecciones, donde la parte más significativa del valor agregado del sector lo representan los productores de fibras naturales como el algodón y las empresas productoras de fibras textiles sintéticas y artificiales donde estas últimas demuestran el altísimo contenido tecnológico de la petroquímica secundaria.

Figura 11: Cadena productiva textil y confecciones

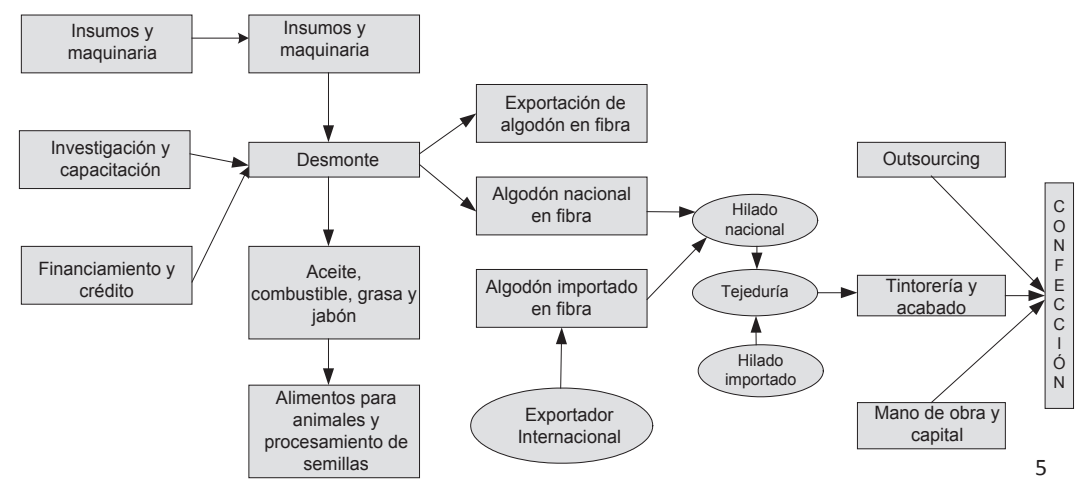

Fuente: MiPymes, clusters y cadenas productivas, Universidad de Lima 2005

\section{Modelos de cadenas productivas del sector servicios}

El sector servicios es tradicionalmente llamado sector terciario de la economía, como su nombre lo indica, propiamente no es productivo porque no produce bienes materiales, aunque incluye todas aquellas actividades necesarias para el funcionamiento de la economía no integrada en los sectores agropecuario e industrial. El sector servicios proporciona atención personal, es decir, servicios que contribuyen a la formación del producto e ingreso nacional.

Las principales ramas o actividades del sector servicios son: comercio, restaurantes y hoteles, transporte, telecomunicaciones, servicios financieros, alquiler de inmuebles y servicios profesionales, entre otros. 
Las cadenas productivas comerciales ya sean: locales, nacionales e internacionales; son un modelo de negocios donde se requiere de factores como: asesoría, outsourcing, plataforma tecnológica, soporte técnico y actualización (NAFIN, 2006).

Figura 12. Cadena productiva comercial (nacional)

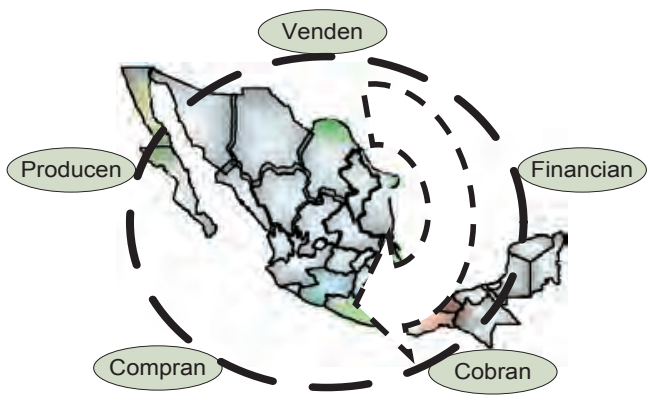

Fuente: Nacional Financiera, 2006

En la Figura 12, se aprecia la cadena productiva comercial donde se proveen de suministros de los productores, venden, se financian, cobran y compran y generan actividades cíclicas. Las empresas privadas del sector comercial que tienen este tipo de cadena productiva son: Liverpool, Muebles Ara, Chedraui, Bimbo, Cemex y Comercial Mexicana, entre otras.

\section{Conclusiones}

1. Existen distintos tipos de cadenas productivas que pueden ser clasificadas con base en su grado de integración: cadena completa, incompleta e integrada.

2. La literatura sobre los modelos de cadenas productivas + puede ser clasificadas de acuerdo a su alcance en: globales y sectoriales. Las cadenas productivas globales de acuerdo con Gereffi (1999), son las cadenas productivas destinadas al productor y al comprador, donde sus características están enraizadas en sectores industriales diferenciados, están dirigidas por diferentes tipos de capital transnacional (industrial y comercial respectivamente). Las cadenas sectoriales varían en sus competencias centrales (en nivel empresa) y sus barreras de entrada (en el nivel sectorial).

3. Las cadenas productivas pueden ser clasificadas también de acuerdo a los tres sectores económicos: agropecuario, industrial y de servicios. Existen, además, cadenas híbridas que abarcan a dos sectores tales como las cadenas 
productivas agroindustriales, donde le dan gran importancia a la tecnología en el acercamiento del productor con el consumidor final. La Tabla 1, muestra el resumen de los tipos y modelos de cadena productiva descritos en este artículo.

Tabla 1: Cuadro de resumen

TIPOS DE CADENA PRODUCTIVA Villacorta

(2005)

a) CADENA COMPLETA

Es una cadena productiva, compuesta por todos los elementos (proveedores de insumos, sistemas productivos, agroindustria, comercialización, mayorista y minorista y consumidores finales).

\section{b) CADENA INCOMPLETA}

Es una cadena productiva con uno o más de los componentes anteriores. c) CADENA INTEGRADA

Es una cadena productiva cuyo producto se constituye en insumo para otra cadena.

\section{MODELOS DE CADENA PRODUCTIVA}

a) GLOBALES Gereffi (1999)

\section{PRODUCTOR}

Las cadenas productivas dirigidas al productor, son aquellas en las que los grandes fabricantes, comúnmente transnacionales, juegan los papeles centrales en la coordinación de redes de producción (incluyendo vínculos hacia atrás y hacia delante).

\section{COMPRADOR}

Las cadenas productivas destinadas al comprador se refiere a aquellas industrias en las que los grandes detallistas, los comercializadores y los fabricantes de marca juegan papeles de pivotes en el establecimiento de redes de producción descentralizada en una variedad de países exportadores, comúnmente localizados en el tercer mundo.

\section{SECTOR AGROPECUARIO}

Los sectores agropecuarios presentan una falta de integración entre los agentes productivos, los cuales por el contrario compiten individualmente entre sí (proveedores de insumos, intermediarios, medios de transformación, comercialización y demás. Los modelos de cadenas productivas de las cuatro actividades que conforman este sector son: (García, 2005).

Cadenas productivas de actividades: agrícolas, ganaderas, silvícolas, pesqueras

\section{SECTOR INDUSTRIAL}

Las cadenas productivas industriales muestran el valor más significativo en la calidad del proceso de transformación del producto y la tecnología así como también en la distribución y comercialización de los productos (Campebell J, Rozemberg, R. y Svarzman, 1999).

\section{SECTOR SERVICIOS}

Las cadenas productivas comerciales ya sean: locales, nacionales e internacionales; son un modelo de negocios donde se requiere de factores como: asesoría, outsourcing, plataforma tecnológica, soporte técnico y actualización (NAFIN, 2006).

\section{AGROINDUSTRIALES}

Los modelos de cadenas productivas agroindustriales tienen relaciones intersectoriales con las actividades agropecuarias e industriales. Las cadenas productivas agroindustriales, demuestran la importancia de la tecnología en el acercamiento del productor al consumidor final, vía tecnología desde el punto de vista de venta, apoyadas fuertemente en tecnologías de información (Programa Estatal de Ciencia y Tecnología del Estado de Jalisco, 2007)

Fuente: Elaboración propia con base en la información documental 
4. Las cadenas productivas permiten analizar sistemas agro-alimentarios, sistemas industriales y también sistemas económicos; por lo que algunos países han desarrollado proyectos de integración productiva enfocados en los temas mencionados, a través de sus gobiernos para construir políticas de desarrollo articuladas y de sus universidades principalmente del área administración de negocios de graduados para elaborar investigaciones de integración regional.

5. Los modelos de cadenas productivas son herramientas de análisis, que nos permiten determinar los puntos fuertes y potenciales de desarrollo para determinar la asociatividad de los elementos que la forman. Con base en la asociatividad y según las relaciones de cooperación-competencia, concentraciones empresariales y alianzas estratégicas que existan en la cadena productiva se puede determinar la existencia de redes, clúster y cadena del valor, que permiten a la cadena productiva desempeñarse competitivamente al maximizar las fuerzas y oportunidades y minimizar las amenazas y debilidades del entorno interno y externo donde se desarrolla la cadena productiva.

6. Para realizar un análisis de cadenas productivas se debe de enfocar en: las relaciones de los actores y las implicaciones para del desarrollo económico.

7. El proceso de formación de una cadena productiva tiene implícito el análisis de la cadena y establecer diálogos de acción, que son los planes de apoyo ó convenios para el fomento de estas.

8. Dentro de la cadena productiva debe de existir cierta autoridad, coordinación o gobierno, que son las relaciones integradas entre los actores que participan en el aparato productivo.

9. Los modelos de cadenas productivas son representaciones de sistemas productivos que integran agentes, actores o conjuntos de empresas y añaden valor agregado a los productos o servicios en cada uno de sus eslabones; la diferencia entre ellos es el propósito al que sirven, se destaca el actor que se pretende priorizar.

10. Uno de los retos teóricos que podría ser objeto de una tesis doctoral es estudiar la evolución de las cadenas productivas en el tiempo de acuerdo las tipología descrita en este trabajo

\section{Referencias}

Bueno, C. (1996). Dirección estratégica de la empresa. Metodología, Técnicas y casos. Madrid: Pirámide 
Campbell J, Rozemberg R. Y Svarzman. (1999). Argentina- Brasil en los 80s: entre la cornisa y la integración. MERCOSUR: entre la realidad y la utopía. Ed. Nuevohacer

Cantú, H. (2001). Desarrollo de una cultura de calidad. México: Mc Graw Hill

CEPAL (2003). Apertura Económica y encadenamientos productivos

Croxton, K, García- Dastugue, S. and Lambert, D. (2001).The supply chain management processes. International Journal of Logistics Management, 12 (2), 13-17

De Castro A, (2002). Productive chain: A conceptual frame for supporting technological prospection. Espacios. Vol. 23 (2) 2002

Diario Oficial de la Federación (2002). Ley para el desarrollo de la competitividad de la micro, pequeña y mediana empresa. México: Decreto Octubre 2002

Dini, M. (2003). Plan operativo para el fomento de los clusters en Perú

García, J. (2005). Pymes, Clusters y Cadenas Productivas. Universidad de Lima Perú. Pag.10-27

Gaona E. Aguirre E. (2007) Las Micro, Pequeñas y Medianas Empresas (Mipymes) Mexicanas y su participación en el comercio exterior Investigadores de la Universidad Autónoma de Hidalgo. Extraído de http://www.eumed.net/eve/ resum/07-enero/egr.htm el 14 de Agosto de 2009

Gereffi, G. (2001). Las cadenas productivas como marco analítico para la globalización. Problemas de Desarrollo, vol. 32. núm. 125. México, IIEc- UNAM, Abril Junio, 2001

Gereffi, G. (1999). International Trade and Industrial Upgrading in the Apparel commodity Chain. Journal of International Economics, Vol.48:37-70

Gereffi, G. y M. Korzenniewicz. (1994). Commodity chains and global capitalism, Westport, CT, Praeger

Gereffi, G. y Wyman, D. (editors), (1990). Manufacturing miracles: parts of industrializations in Latin America and East Asia, Princeton, NJ. Princeton University Press

Gomes De Castro A. (1999). Guía para la elaboración y estudio de cadenas productivas locales. EMBRAPA Bolivia

Grantt, R. (2005). Dirección estratégica. Conceptos, técnicas y aplicaciones. Civitas, Madrid 
Guitart, L. (2005). La ruptura de la cadena de valor como consecuencia de la subcontratación. Investigación cualitativa mediante el análisis de casos. Universidad de Barcelona

Humphrey J. y H. Schmitz (2000). Governance and Upgrading: Linking industrial cluster and Global Value Chain Research. Documento de trabajo No. 120 del Institute of Development Studies Brighton: University of Sussex

Jiménez, J. (2002). Análisis de la cadena de valor de 4 empresas medianas del sector eléctrico en México D.F. y su área metropolitana. Tesis para obtener el grado de maestro en ciencias por el Instituto Politécnico Nacional, Escuela Superior de Comercio y Administración, Sto. Tomás, sección de estudios de posgrado e investigación México, D.F.

Kaplinsky, R. (2000). Spreanding the Gains from Globalization: What can be learned value chain analisis. Documento de trabajo de IDS No. 110. Institute of Development Studies, Brighton: University of Sussex

Laura, M. (2006). Cadenas productivas conglomerados y redes: el caso de las MIPYMES Peruanas. Comisión de promoción de la pequeña y micro empresa, ministerio de trabajo y promoción del empleo del gobierno de Perú

Morales, J. (1999). Diseño de un modelo de negocios basado en E- Comerce para las PYMES, Mexicanas. Tesis para obtener el grado de maestro en ciencias por el Instituto Politécnico Nacional, Escuela Superior de Comercio y Administración, Sto. Tomás, sección de estudios de posgrado e investigación México, D.F.

Pierce, Leslie. (2003). Cadenas productivas: Una alternativa para afrontar la integración nacional. Año 8 no.14 junio 2003. pág. 117. Escuela Superior de Negocios para Graduados. Perú

Porter. M. (1999). Ser competitivo - nuevas aportaciones y conclusiones. México: Deustos S.A.

Porter, M. (1986). Ventaja competitiva-creación y sostenimiento de un desempeño superior. Ed. Continental. México

Programa Estatal de Ciencia y Tecnología del Estado de Jalisco, México. (2007)

Ramírez, P. (1997). Contabilidad Administrativa. México: Continental

Spens, K, and Bask, A. (2002). Developing a framework for supply chain management. The international journal of Logistics Management, 13 (1), 73-88

Universidad Agraria La Molina de Perú, (2005). Cadenas Productivas en el Sector Pecuario. Publicada el día viernes, 11 de Noviembre 2005. Perú Ministerio de Agricultura. Extraído de http://www.lamolina.edu.pe/proyeccion/oaeps/noticias/ detalledenoticial el 13 de Julio de 2008 
Van Der, Heyden D, Camacho, P. (2006). Guía metodológica para el análisis de cadenas productivas. Centro Internacional para el desarrollo, Lima Perú

Villacorta I. (2005). Guía para la elaboración de estudios de cadenas productivas locales. PADER- COSUDE. Bolivia, p 6-26

Wood, A. (2001). Value Chains: An Economist's Perspective. IDS Bulletin, Número especial: The Value of Values Chains, Vol.32, No.3 41-45 